\title{
Novel Homodimer Metabolites of GDC-0994 via Cytochrome P450-Catalyzed Radical Coupling ${ }^{\text {[ }}$
}

\author{
(1) Ryan H. Takahashi, ${ }^{1,2}$ Jessica M. Grandner, ${ }^{2}$ Sudheer Bobba, ${ }^{2}$ Yanzhou Liu, Paul Beroza, \\ Donglu Zhang, and Shuguang Ma
}

Drug Metabolism and Pharmacokinetics (R.H.T., S.B., D.Z., S.M.) and Discovery Chemistry (J.M.G., Y.L., P.B.), Genentech, Inc., South San Francisco, California

Received November 20, 2019; accepted March 4, 2020

\begin{abstract}
Two novel homodimer metabolites were identified in rat samples collected during the in vivo study of GDC-0994. In this study, we investigated the mechanism of the formation of these metabolites. We generated and isolated the dimer metabolites using a biomimetic oxidation system for NMR structure elucidation to identify a symmetric dimer formed via carbon-carbon bond between two pyrazoles and an asymmetric dimer formed via an aminopyrazole-nitrogen to pyrazolecarbon bond. In vitro experiments demonstrated formation of these dimers was catalyzed by cytochrome P450 enzymes (P450s) with CYP3A4/5 being the most efficient. Using density functional theory, we determined these metabolites share a mechanism of formation, initiated by an $\mathrm{N}-\mathrm{H}$ hydrogen atom abstraction by the catalytically active iron-oxo of P450s. Molecular modeling studies also show these dimer metabolites fit in the CYP3A4 binding
\end{abstract}

site in low energy conformations with minimal protein rearrangement. Collectively, the results of these experiments suggest that formation of these two homodimer metabolites is mediated by CYP3A, likely involving activation of two GDC-0994 molecules by a single P450 enzyme and proceeding through a radical coupling mechanism.

\section{SIGNIFICANCE STATEMENT}

These studies identified structures and enzymology for two distinct homodimer metabolites and indicate a novel biotransformation reaction mediated by CYP3A. In it, two molecules may bind within the active site and combine through radical coupling. The mechanism of dimerization was elucidated using density functional theory computations and supported by molecular modeling.

\section{Introduction}

GDC-0994 (structure shown in Fig. 1A) is a selective, potent, orally bioavailable, small molecule inhibitor of extracellular signal-regulated kinase (ERK)1 and ERK2. It has proven antitumor activity in RAS- and RAF-mutant human tumor cell-line models in vitro and in vivo (Blake et al., 2016). Based on its mechanism of action and the high frequency of mitogenactivated protein kinase pathway activation in human cancers, GDC-0994 was in development as an anticancer therapeutic with potential in a broad array of cancer indications (Ren et al., 2015; Kirouac et al., 2017) including its clinical testing in patients with locally advanced or metastatic solid tumors (Varga et al., 2020). While thoroughly evaluating the metabolic profile of GDC-0994 in rats, the nonclinical toxicological species that supported entry into clinical development, we identified two novel dimer metabolites (M13 and M14) that formed via cytochrome $\mathrm{P} 450$ (P450) oxidation.

$\mathrm{P} 450$ enzymes catalyze a wide range of reactions for the metabolism of xenobiotics. The most common reactions include carbon hydroxylation, heteroatom oxygenation, dealkylation, and epoxidation (Ortiz de Montellano and De Voss, 2005). However, a variety of uncommon reactions by $\mathrm{P} 450 \mathrm{~s}$ have been observed. These reactions are of particular interest because their products are often not easily predicted (Guengerich and Munro, 2013).

${ }^{1}$ Current affiliation: Drug Metabolism and Pharmacokinetics, Denali Therapeutics, South San Francisco, California.

${ }^{2}$ R.H.T., J.M.G., and S.B. contributed equally to this work.

https://doi.org/10.1124/dmd.119.090019.

S This article has supplemental material available at dmd.aspetjournals.org.
From a research perspective, these case examples provide interesting opportunities to probe the diversity of $\mathrm{P} 450$ function. From a drug discovery and development perspective, an unexpected metabolite adds to uncertainties and associated risks of a candidate molecule upon its first administration to human subjects (Schadt et al., 2018). Therefore, understanding reaction mechanisms, structural requirements, and specific isoform(s) responsible for these reactions is critical to anticipate and mitigate metabolic liabilities.

Of the P450 isoforms found in humans, CYP3A4 is most frequently identified as the principalmetabolizing enzyme of approved drugs (Cerny, 2016). CYP3A4 has a large and flexible active site that accommodates a variety of ligands, including large substrates such as cyclosporine A (molar mass $1203 \mathrm{~g} / \mathrm{mol}$ ). Understanding the diversity of reactions that CYP3A4 can catalyze provides a foundation to understand the breadth of possible metabolic products it can generate and feed back to the design and optimization of new molecular entities.

In this report, we describe the identification and structure determination of two distinct homodimers of GDC-0994 and provide in vitro evidence that CYP3A catalyzed their formations. We also embarked on a computational study using density functional theory (DFT) to determine the likely mechanism of dimer formation and molecular modeling to evaluate the feasibility of dimer formation within CYP3A4.

\section{Materials and Methods}

Chemicals and Reagents. $\left[{ }^{14} \mathrm{C}\right] \mathrm{GDC}-0994$ was synthesized by Selcia Limited (Essex, UK) with radiochemical purity $>98.5 \%$ (specific activity $52.23 \mathrm{mCi} / \mathrm{mmol}$ ), and nonradiolabeled GDC-0994 was synthesized at Genentech 
A
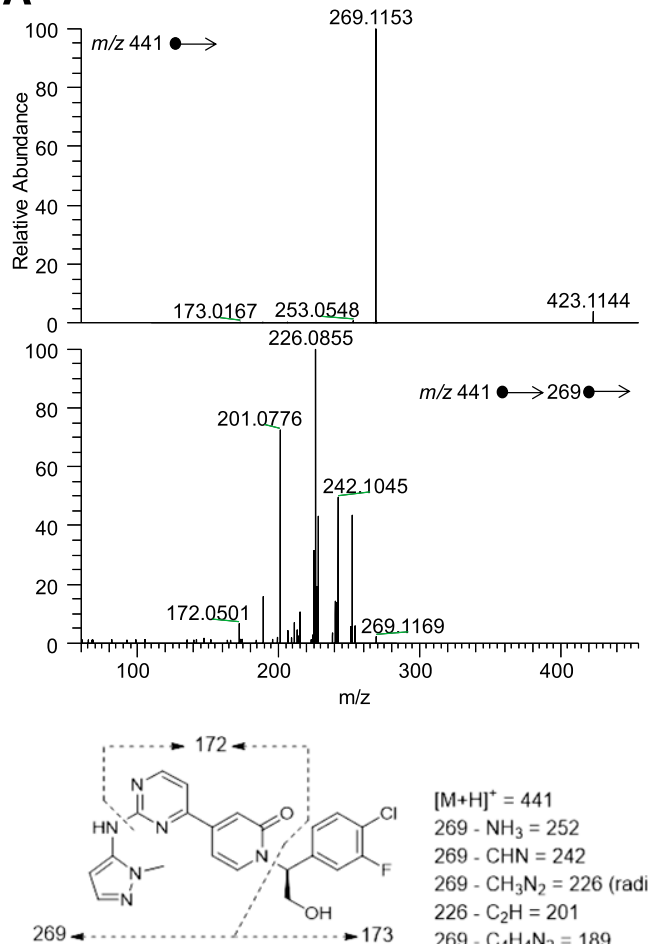

$[\mathrm{M}+\mathrm{H}]^{+}=441$

$269-\mathrm{NH}_{3}=252$ 269. $\mathrm{CHN}=242$ $226-\mathrm{C}_{2} \mathrm{H}=201$
$269-\mathrm{CH}_{3} \mathrm{~N}_{2}=226$ (radical) $269-\mathrm{C}_{4} \mathrm{H}_{4} \mathrm{~N}_{2}=189$

C
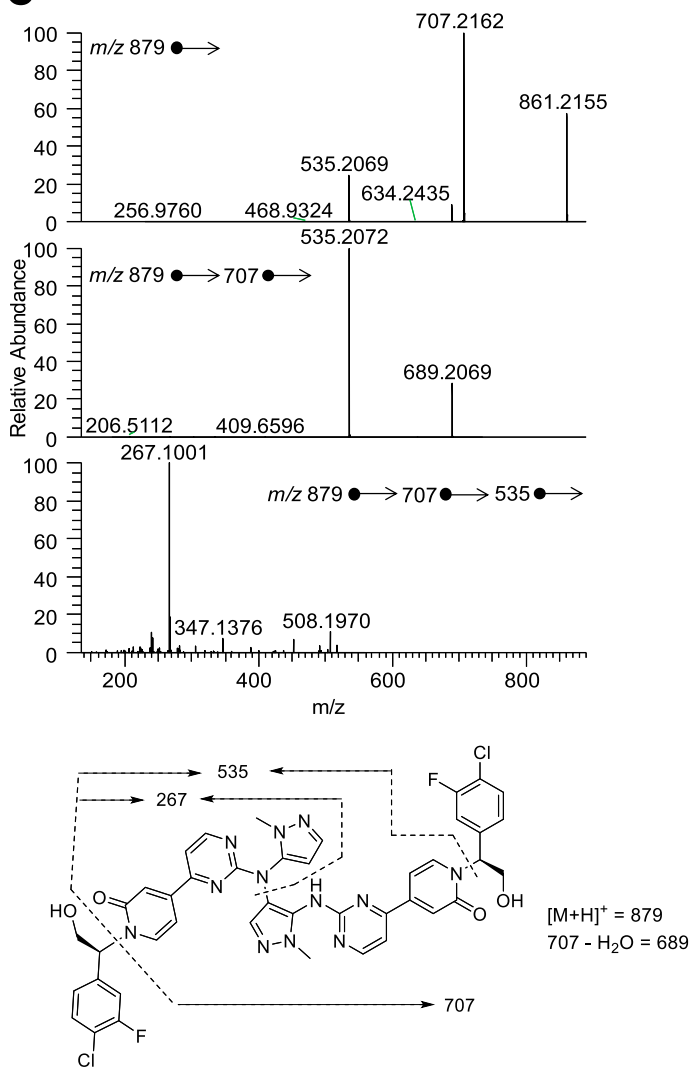

Fig. 1. $\mathrm{MS}^{2}$ and $\mathrm{MS}^{\mathrm{n}}$ product ion spectra of (A) GDC-0994, (B) M13, and (C) M14

\section{B}
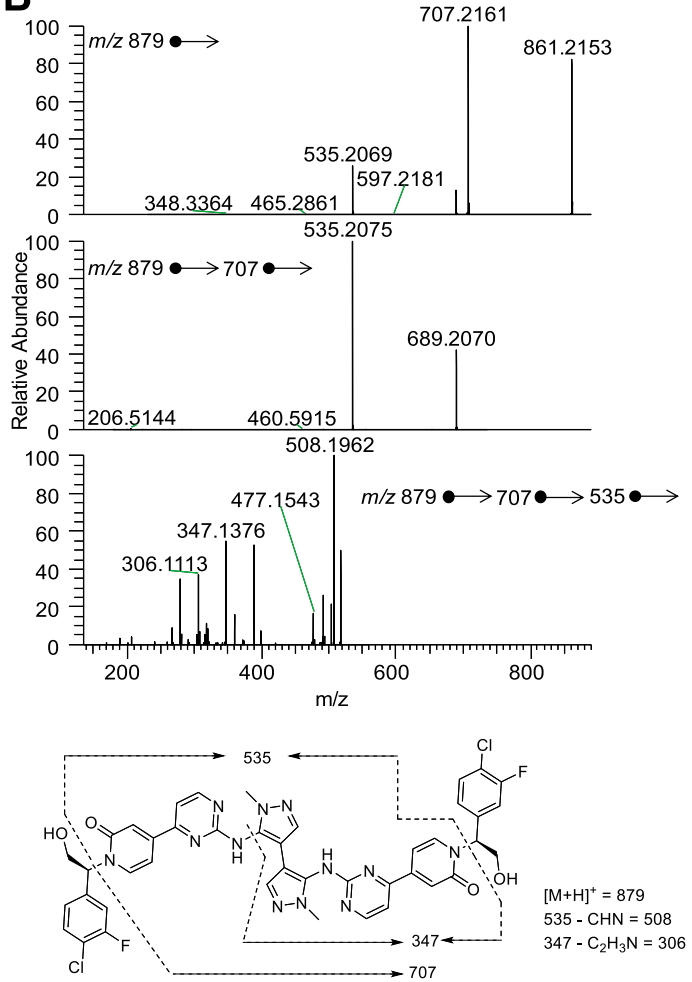
$535-\mathrm{CHN}=508$
$347-\mathrm{C}_{2} \mathrm{H}_{3} \mathrm{~N}=306$ 
(South San Francisco, CA). Liver microsomes and recombinant P450 enzymes were purchased from BD Gentest (San Jose, CA) or Bioreclamation IVT (Westbury, NY). Horseradish peroxidase was obtained from Sigma-Aldrich (St Louis, MO). Myeloperoxidase from human leukocytes was purchased from EMD Millipore Co. (Temecula, CA). All chemical reagents and solvents were purchased from commercial sources at the highest purities possible.

In Vivo Metabolite Identification Study. Male and female Sprague-Dawley rats were orally administered a single dose of $\left[{ }^{14} \mathrm{C}\right]$ GDC-0994 at $50 \mathrm{mg} / \mathrm{kg}(100$ $\mu \mathrm{Ci} / \mathrm{kg}$ ) in $1 \%$ carboxymethylcellulose, $0.5 \%$ Tween, and $5 \mathrm{mM}$ citrate solution. In vivo study design, sample collection, and radioanalysis are described in Supplemental Materials. Metabolite profiling and identification was performed using liquid chromatography coupled with simultaneous radiodetection and highresolution mass spectrometric analysis. Method details are also provided in Supplemental Materials.

Metabolite Generation Using Biomimetic Enzymes. BMO Production Kit (HCK1001-03-027-A7-10) (HepatoChem Inc., Beverly, MA) was used to generate, scale up, and isolate two metabolites of interest, M13 and M14. Standard protocols provided by the vendor were followed for screening, optimization, and production of metabolites. The incubations were performed with $6.25 \mathrm{mM}$ of GDC-0994 free base. The dimer metabolites were purified by semipreparative liquid chromatography using a Hypersil Gold C18 column (150 $\times 10 \mathrm{~mm}, 5 \mu \mathrm{m}$ particle size; Thermo Scientific, San Jose, CA) with mobile phases of $0.1 \%$ formic acid in water and acetonitrile at a constant flow rate of $4.6 \mathrm{ml} / \mathrm{min}$. The incubation products were separated by gradient elution, and fractions were collected and surveyed by liquid chromatography-mass spectrometry for the analytes of interest. The fractions containing M13 and M14 were then separately combined and evaporated to dryness under vacuum.

Structure Determination by NMR. All NMR experiments were collected on an Avance II spectrometer (Bruker BioSpin, Billerica, MA) operating at $500 \mathrm{MHz}$ for ${ }^{1} \mathrm{H}, 125 \mathrm{MHz}$ for ${ }^{13} \mathrm{C}$ equipped with $5 \mathrm{~mm}$ TCI cryoprobe with Z-gradient. Each metabolite sample was dissolved in $180 \mu$ l of DMSO- $d 6$ (Cambridge Isotope Laboratories, Tewksbury, MA). One-dimensional ${ }^{1} \mathrm{H}$ and ${ }^{13} \mathrm{C}$ NMR were collected at ambient temperature and $335 \mathrm{~K}$, respectively. Chemical shifts were referenced to tetramethylsilane at $\delta=0 \mathrm{ppm}$. Two-dimensional correlation spectroscopy, heteronuclear single quantum correlation spectroscopy, heteronuclear multiple bond correlation spectroscopy, and rotating frame nuclear Overhauser effect spectroscopy were collected at $335 \mathrm{~K}$.

In Vitro Metabolism. GDC-0994 $(10 \mu \mathrm{M})$ was incubated with liver microsomes (male rat, female mouse, male beagle dog, male cynomolgus monkey, or 200 mixed sex donor pooled human, $0.5 \mathrm{mg} / \mathrm{ml}$ ) in potassium phosphate buffer (100 mM, pH 7.4) containing magnesium chloride ( $3 \mathrm{mM})$ supplemented with $1 \mathrm{mM}$ NADPH in an incubation volume of $0.5 \mathrm{ml}$. Incubations with individually expressed recombinant P450 enzymes (Supersomes) were completed similarly with GDC-0994 $(1 \mu \mathrm{M})$ and a constant amount of P450 (40 pmol/ml). Inhibition experiments were conducted with human liver microsomes using standard concentrations of $\mathrm{P} 450$ isoformselective chemical inhibitors: 1-aminobenzotriazole (ABT; $1 \mathrm{mM}$, panP450), furafylline (10 $\mu \mathrm{M}, \mathrm{CYP} 1 \mathrm{~A} 2)$, 2-phenyl-2-(1-piperidinyl)propane (20 $\mu \mathrm{M}$, CYP2B6), montelukast ( $2 \mu \mathrm{M}, \mathrm{CYP} 2 \mathrm{C} 8)$, sulfaphenazole $(10 \mu \mathrm{M}$, CYP2C9), 3-benzylnirvan (2 $\mu \mathrm{M}, \mathrm{CYP} 2 \mathrm{C} 19)$, quinidine (1 $\mu \mathrm{M}, \mathrm{CYP} 2 \mathrm{D} 6)$, ketoconazole (1 $\mu \mathrm{M}, \mathrm{CYP} 3 \mathrm{~A} 4)$, or troleandomycin $(20 \mu \mathrm{M}, \mathrm{CYP} 3 \mathrm{~A} 4)$. In the incubations with $\mathrm{ABT}$ and troleandomycin, human liver microsomes were preincubated with inhibitors for 15 minutes before GDC-0994 was added to the incubations. At the end of incubation, reactions were stopped, and proteins were removed by adding acetonitrile and centrifuging.

GDC-0994 $(100 \mu \mathrm{M})$ was incubated with horseradish peroxidase $(40 \mathrm{U} / \mathrm{ml})$, $\mathrm{NaCl}(150 \mathrm{mM})$, and $\mathrm{H}_{2} \mathrm{O}_{2}(100 \mu \mathrm{M})$ in $100 \mathrm{mM}$ phosphate buffer $\mathrm{pH} 7.4$ at $37^{\circ} \mathrm{C}$ for 1 hour. GDC-0994 $(100 \mu \mathrm{M})$ was separately incubated with myeloperoxidase $(0.1 \mathrm{mg} / \mathrm{ml}), \mathrm{NaCl}(150 \mathrm{mM})$, and $\mathrm{H}_{2} \mathrm{O}_{2}(100 \mu \mathrm{M})$ in $100 \mathrm{mM}$ phosphate buffer $\mathrm{pH} 6.0$ at $37^{\circ} \mathrm{C}$ for 1 hour. Reactions were quenched with three volumes of acetonitrile. The supernatant was diluted with three volumes of water and injected for liquid chromatography-mass spectrometry analysis.

Computational Methods. All DFT computations were performed using Gaussian09 (Frisch et al., 2013). Geometry optimizations and frequency calculations were performed at the B3LYP (Becke, 1993) level using LANL2DZ for Fe and 6-31G(d) on all other atoms. Frequencies were used to check for the presence of a local minimum (zero negative frequencies) or a transition structure (exactly one negative frequency, corresponding to the desired transformation).
Frequencies were also used to compute enthalpy and free energy corrections at a standard state of $1 \mathrm{~atm}$ and $298.15 \mathrm{~K}$. An additional correction for entropy was used to apply a quasiharmonic approximation, as discussed by Truhlar (Zhao and Truhlar, 2008; Ribeiro et al., 2011). Single point energy calculations were computed for each structure at the B3LYP-D3(BJ) (Grimme et al., 2011) level using LANL2DZ for iron and $6-311+\mathrm{G}(\mathrm{d}, \mathrm{p})$ on all other atoms and the SMD (Marenich et al., 2009) implicit solvent models for water.

Molecular Modeling. Molecular modeling studies were performed using MOE (Chemical Computing Group ULC, 2018) with the MMFF94x forcefield (Halgren, 1996a,b,c,d; Halgren and Nachbar, 1996; Halgren, 1999a,b), and docking was performed with Glide (Schrödinger LLC, 2016). Conformational analyses of the dimer structures were performed with the sdfMMConfAnalysis module of the Chemalot software package (Lee et al., 2017).

\section{Results}

Identification of Metabolites. GDC-0994 was extensively metabolized in rats (Supplemental Fig. 1). A total of 14 metabolites were tentatively identified (Supplemental Fig. 2), primarily consisting of oxidation, $N$-dealkylation, glucuronidation, or a combination of these reactions. Two distinct radiopeaks, M13 and M14, which eluted later than unchanged GDC-0994, were observed in bile and feces profiles (Supplemental Fig. 1). The protonated molecular ions observed in M13 and M14 were identical $\left[\mathrm{m} / \mathrm{z} \quad 879.2343, \mathrm{C}_{42} \mathrm{H}_{35} \mathrm{~N}_{12} \mathrm{O}_{4} \mathrm{Cl}_{2} \mathrm{~F}_{2}(-0.1\right.$ ppm)], which corresponded to two molecules of GDC-0994 connected by loss of two hydrogens, suggesting they are homodimer metabolites. The $\mathrm{MS}^{2}$ and $\mathrm{MS}^{\mathrm{n}}$ product ion spectra of GDC-0994, M13, and M14 are shown in Fig. 1. M13 and M14 shared highly similar MS ${ }^{2}$ and MS ${ }^{3}$ fragmentation spectra (Fig. 1, B and C), which showed product ions at $m / z 707$ (loss of chloro fluoro phenyl ethanol), $m / z 689$ (loss of water from $m / z, 707$ ), and $m / z, 535$ (loss of chloro fluoro phenyl ethanol from $m /$ $z$ 707). However, their $\mathrm{MS}^{4}$ spectra were dramatically different, implying the connectivity of the dimers was the structural distinction between M13 and M14. Heterodimers composed of 1 molecule of GDC0994 and 1 molecule of the $N$-desmethyl metabolite (M12) were only detected in bile by mass spectrometry in significantly lower quantities than M13 or M14. Homodimers of M12 were not detected in any matrices, presumably due to its low overall concentration.

Dimer Metabolite Generation and Structure Elucidation. To fully characterize the structures of the proposed GDC-0994 dimers, we attempted to scale up the metabolites with various in vitro systems. The product yields in microsomal or expressed P450 incubations were too low to efficiently obtain sufficient amounts of product. Thus, metabolite generation was surveyed and scaled using organometallic catalysis purchased from HepatoChem. This tool provides biomimetic catalysts to mimic the suite of $\mathrm{P} 450$ enzymes present in human hepatocytes, offering the researcher a unique approach to quickly find reaction conditions that generate target drug metabolites and scale them to provide sufficient quantities for structural characterization or pharmacological potency testing (Chen and White, 2007; Cusack et al., 2013). From the biomimetic screening panel, M13 and M14 were generated to greatest extent by metalloporphyrin FeTDCIPP and $\mathrm{H}_{2} \mathrm{O}_{2}$ as oxidant. This catalytic system was used to generate sufficient quantities of metabolites for isolation by liquid chromatography and fractionation for definitive structure characterization by NMR.

Based on NMR studies, M13 is a symmetrical dimer, whereas M14 is an asymmetric dimer of GDC-0994 (Fig. 2). At room temperature, proton NMR spectra of both M13 and M14 showed broad resonances due to restricted rotation. Thus, both M13 and M14 were heated to $335 \mathrm{~K}$ to obtain well resolved one-dimensional proton NMR and twodimensional data. Proton NMR of M13 gave only one set of resonances, which was comparable to those observed for GDC-0994. The distinct differences were that one pyrazole proton $\mathrm{H} 16$ at 6.3 ppm was missing 

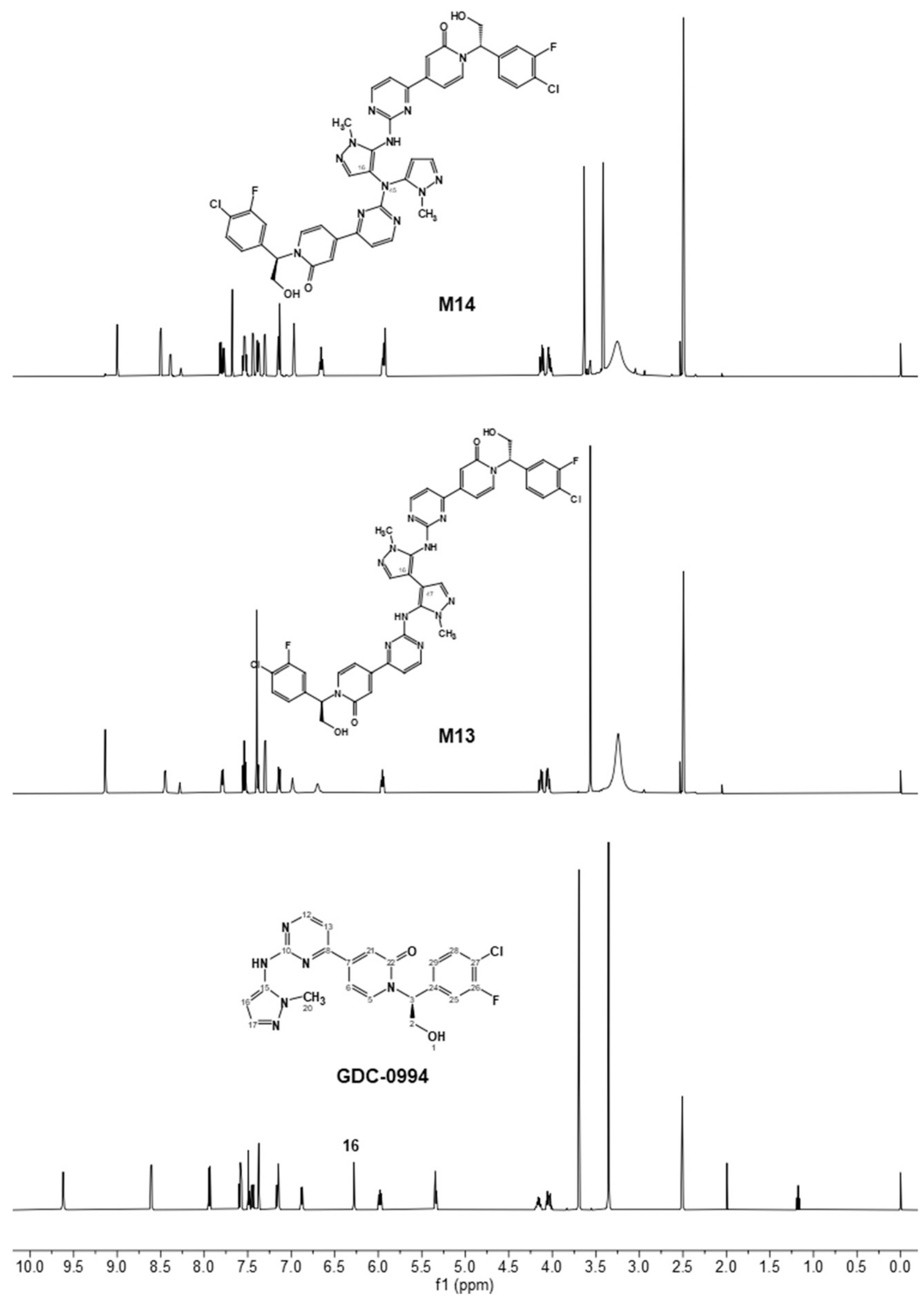

Fig. 2. Proton nuclear magnetic resonance spectroscopy data of GDC-0994 and its homodimer metabolites M13 and M14. 

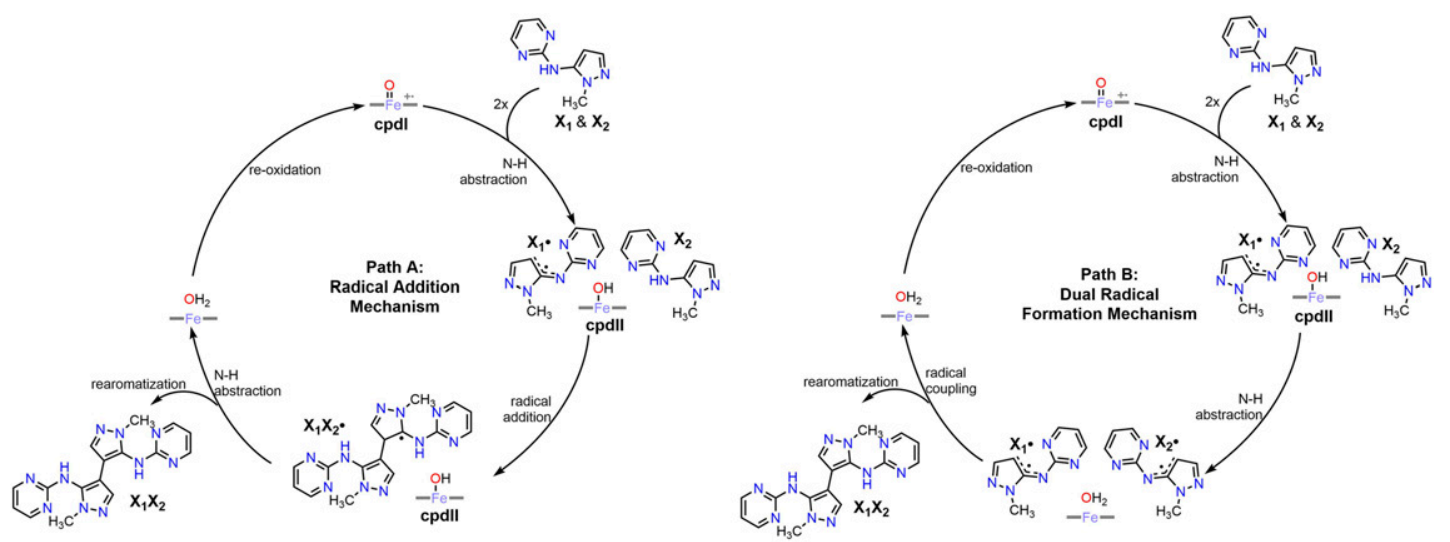

Fig. 3. Possible catalytic cycles for formation of C-C dimer and C-N dimer (only formation of C-C dimer is shown for simplicity). Pathway A, left; Pathway B, right. X (X1 and X2) is a small model of GDC-0994 used for computational purposes.

and the other pyrazole proton $\mathrm{H} 17$ became singlet by losing coupling with H16. There was also an extra quaternary carbon on carbon-13 NMR of M13. These structural features were consistent with two GDC-0994 molecules forming a symmetrical dimer at $\mathrm{C} 16$ position. The new carbon-carbon bond formed in M13 was labeled as C16-C47. For M14, proton NMR gave two sets of resonances consistent with GDC-0994 with two resonances missing, one pyrazole proton H16 from one GDC0994 molecule, and H45 from N-H of another GDC-0994 molecule. This evidence concludes that a carbon-nitrogen bond was formed between two GDC-0994 molecules to give an asymmetric dimer with same molecular weight as M13. The new carbon-nitrogen bond formed in M14 was labeled as C16-N45.

In Vitro Formation of Dimer Metabolites. In vitro studies demonstrated dimer formation with liver microsomal incubations for rats, mice, rabbits, dogs, monkeys, and humans, albeit all to low extent. With human liver microsomes, the formations of the dimers were time and NADPH dependent and were abolished when P450 enzymes were inactivated by preincubating with ABT or ketoconazole, pan-P450 and CYP3A inhibitors, respectively (Supplemental Fig. 3). Incubations with singly expressed recombinant P450s showed CYP3A4 and 3A5 were the most productive in generating M13 and M14.

Incubations of GDC-0994 with horseradish peroxidase in the presence of $\mathrm{Cl}^{-}$and $\mathrm{H}_{2} \mathrm{O}_{2}$ produced both $\mathrm{M} 13$ and M14 with less than $5 \%$ yield (Supplemental Fig. 4), providing evidence of a radical initiation of the mechanism. Dimers were not detected in the incubations of GDC-0994 with myeloperoxidase.

Mechanism of Dimer Metabolite Formation. Density functional theory was used to computationally evaluate two mechanisms of dimer formation (Fig. 3, Pathways A and B), using a truncated model of GDC0994 (X). Computations show that the preferred mechanism (Fig. 4) is Pathway B, involving dual radical formation followed by radical coupling. Pathway A is disfavored due to the high energetics of radical addition to a neutral monomer (Fig. 5).

Docking of either dimer product (M13 or M14) to CYP3A4 (Protein Data Bank code 6BD5) did not yield reasonable substrate poses. Suspecting the protein conformation captured in the crystal structure does not adequately capture the flexibility of the binding pocket, we built custom models for each dimer-protein complex using a protocol similar

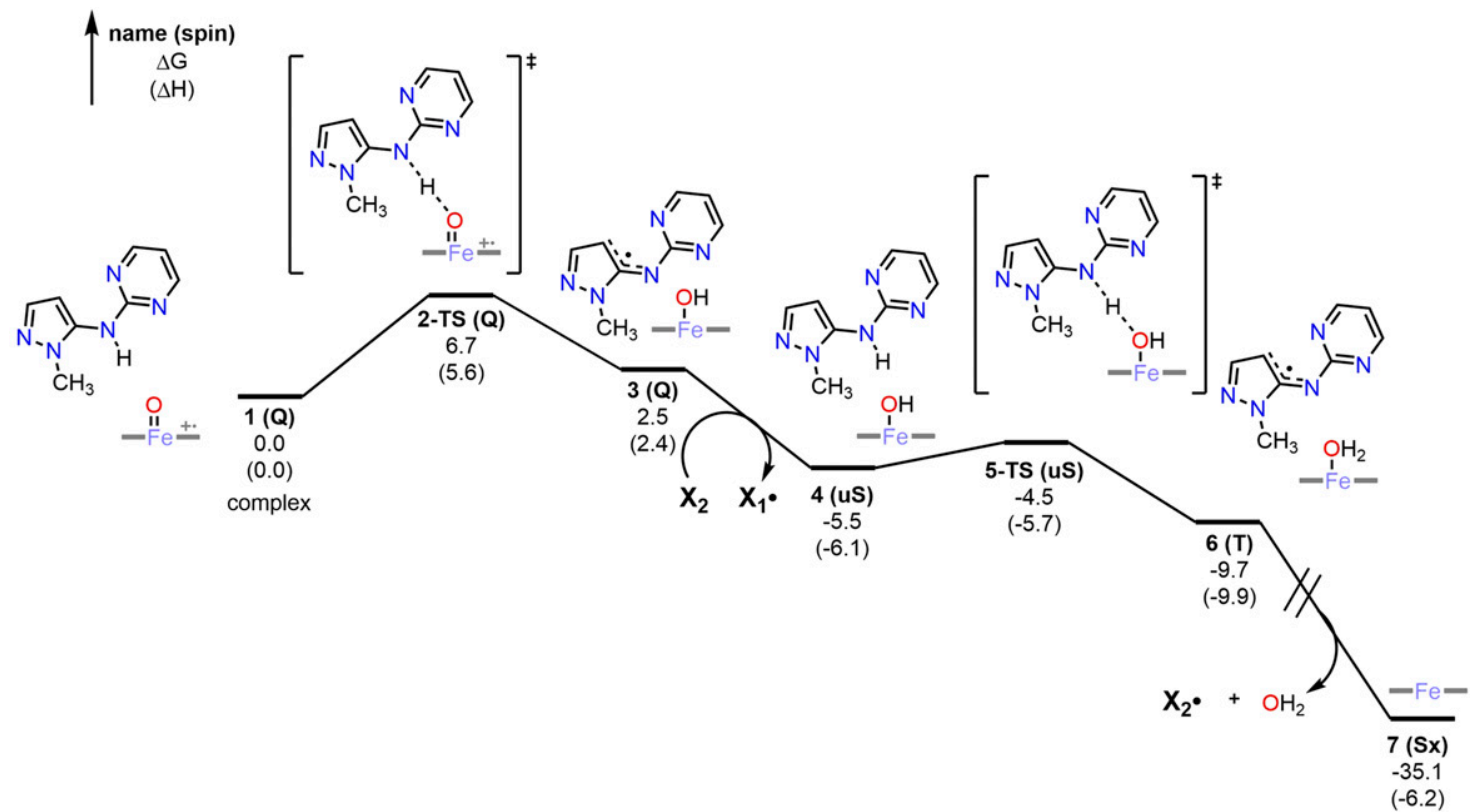

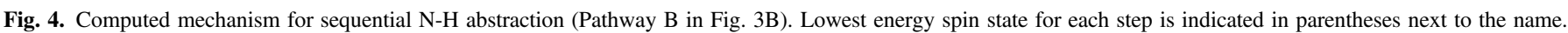
Spin state abbreviations: Q, quartet; Sx, sextet; T, triplet; uS, open-shell singlet. 


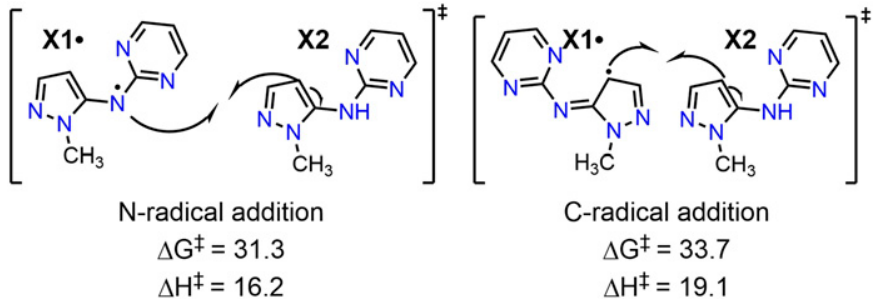

Fig. 5. Barriers to N-radical addition (left) and C-radical addition (right).

to that described by Hayes et al. (2014). Binding pocket residues of the enzyme were mutated to alanine (details in Supplemental Information), and the core of each dimer structure $\left(\mathbf{X}_{1} \mathbf{X}_{2}\right.$ in Fig. 3 and the N-linked dimer analog) were positioned such that the dimer bond was in proximity of the iron-heme. We then gradually and iteratively grew each ligand to its full form in the active site by: 1) adding compound substituents to the core in conformations that avoided steric clashes with the backbone and mutated residues of the protein, 2) minimizing the resulting ligand in situ, 3) removing the protein and minimizing the ligand in vacuo to remove ligand strain, and 4) reintroducing the protein and minimizing side chains and backbone with harmonic constraints in the presence of the fixed ligand. After the dimer structures were completely assembled, the mutated sidechains were restored to the native structure in rotamer states that accommodated the dimer-protein complex. Lastly, each completed and restored complex was minimized one additional time to produce the final models (Fig. 6). The final models had minor differences from the original PDBs, with root mean square deviation values of 1.1 and $0.48 \AA$ for the CYP3A4-M13 complex and CYP3A4M14 complex, respectively. Both M13 and M14 are in low energy conformational states within the complexes $(<2 \mathrm{kcal} / \mathrm{mol}$ from the conformational global energy minimum), so ligand strain is minimal.

\section{Discussion}

The P450-mediated bioactivation of a small molecule to generate a radical reactive intermediate and initiate dimerization was an unexpected biotransformation route. Some preceding examples of similar reactions have been described in the literature, and diverse mechanistic routes for dimer formation have been proposed. Gillam and coworkers reported P450-mediated oxidation of indole to indoxyl, which was subsequently oxidized and dimerized to form indigoids (Gillam et al., 2000; Isin and Guengerich, 2007). Two diastereoisomeric thiophene $S$-oxide dimers were observed in thiophene metabolism in vitro and in vivo in rats, where the combination of molecules was rationalized to occur via a Diels-Alder reaction (Treiber et al., 1997). Similar dimerization was reported for ticlodipine (Dansette et al., 2005).
Dimerization through free radical intermediates has been described for capsaicin and CJ-047710 (Emoto et al., 2007; Reilly et al., 2013). Homodimerization of raloxifene was also reported to occur via a CYP3A4-mediated oxidative reaction (Davis et al., 2011). Several overlaps exist in the observations for CJ-047710, raloxifene, and GDC0994, suggesting these dimerization reactions may share common mechanistic features. After identifying that the dimers of GDC-0994 were formed via CYP3A4 using standard in vitro techniques, we sought to determine the mechanism of their formation to glean a deeper understanding of how these and other dimers are formed.

Two possible mechanisms for the formation of the observed dimers were evaluated with DFT (Pathways A and B, Fig. 3). Aromatic C-H abstraction is not a part of either proposed mechanism. C-H hydrogen abstraction from pyrazole was ruled unfavorable given the high bond dissociation energies of the $\mathrm{C}-\mathrm{H}$ bonds, which were previously computed to be between 118.7 and $122.2 \mathrm{kcal} / \mathrm{mol}$ (Feng et al., 2003). In contrast, the bond dissociation energy for aniline $\mathrm{N}-\mathrm{H}$ has been computed to be significantly lower at $91.4 \mathrm{kcal} / \mathrm{mol}$ (Song et al., 2003). The proposed mechanisms in Fig. 3 are consistent with existing precedence for P450-mediated aromatic couplings (Isin and Guengerich, 2007; Makino et al., 2007; Woithe et al., 2007; Belin et al., 2009; Grandner et al., 2016).

Both Pathways A and B initiate with $\mathrm{N}-\mathrm{H}$ abstraction from model substrate $\mathbf{X}$ by the iron-oxo complex for $\mathrm{P} 450$ (cpd I) to provide a radical species $\left(\mathbf{X}_{\mathbf{1}}\right)$ and iron-hydroxo (cpd II). This radical species $\left(\mathbf{X}_{\mathbf{1}}\right)$ is delocalized between the nitrogen and other atoms in conjugated pyrazole via resonance, and thus the radical can form a new bond from the initial nitrogen involved in the abstraction or carbon in the pyrazole ring. For subsequent steps in Fig. 3, only C-C coupling is shown for simplicity, but $\mathrm{C}-\mathrm{N}$ coupling is proposed to occur in the same manner. In Pathway A, radical addition of $\mathbf{X}_{1}$ to $\mathbf{X}_{2}$ occurs, and subsequent N-H abstraction by cpd II from $\mathbf{X}_{\mathbf{1}} \mathbf{X}_{\mathbf{2}}$ forms the final product. The alternate Pathway B describes forming a second radical species $\left(\mathbf{X}_{\mathbf{2}}\right)$ via $\mathrm{N}-\mathrm{H}$ abstraction, facilitated by cpd II, and radical coupling of $\mathbf{X}_{\mathbf{1}}$ and $\mathbf{X}_{\mathbf{2}}$ for the formation of the final dimer product. Thus, Pathways A and B both describe two hydrogen abstraction steps and a coupling reaction but only diverge in the order of the reaction steps.

The proposed mechanisms were computed using DFT and compared to determine the intrinsically favored pathway (Fig. 4). N-H abstraction by cpd I from $\mathbf{X}_{\mathbf{1}}$ has a barrier of only $6.7 \mathrm{kcal} / \mathrm{mol}$ (2-TS, Fig. 4). This very low barrier indicates abstraction would occur rapidly at room temperature upon appropriate substrate binding. For radical additions of $\mathbf{X}_{\mathbf{1}}$ to ground-state $\mathbf{X}_{\mathbf{2}}$ (Pathway A) to form either C-N or C-C coupled product are $>30 \mathrm{kcal} / \mathrm{mol}$ and prohibitively high at room temperature (Fig. 5). Accounting for reduced entropic costs due to precomplexation of $\mathbf{X}_{\mathbf{1}}$ and $\mathbf{X}_{\mathbf{2}}$ to CYP3A, this barrier is still rather high and indicates
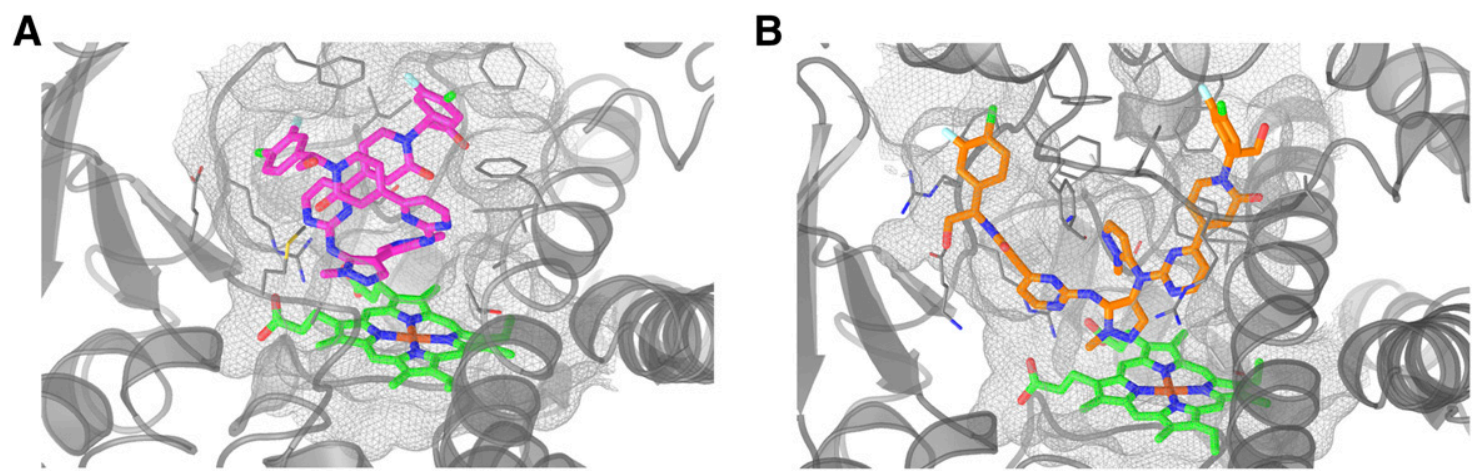

Fig. 6. Molecular models of dimer metabolites M13 (pink, left) and M14 (orange, right) in complex with CYP3A4 (protein and surface in gray, iron-heme in green). 
a disfavoring of this radical addition. In the subsequent steps for Pathway B (Fig. 4), ligand exchange to allow the N-H of $\mathbf{X}_{2}$ to coordinate to the iron-hydroxo cpd II, formed by the first abstraction, is favored by $>5$ $\mathrm{kcal} / \mathrm{mol}$ (3 to 4, Fig. 4). N-H abstraction from $\mathbf{X}_{\mathbf{2}}$ by cpd II is nearly barrierless at $1 \mathrm{kcal} / \mathrm{mol}$ ( 4 to 5-TS, Fig. 4). This barrier is low due to the release of water in the transition state. Radical combination of the delocalized radicals after formation via the two hydrogen abstractions would be rapid, further facilitated by the forced close proximity in the active site. The mechanism is the same for each metabolite, as formation of the two distinct products is facilitated by the delocalized nature of the radicals formed from $\mathrm{N}-\mathrm{H}$ abstraction $\left(\mathbf{X}_{\mathbf{1}}\right.$ and $\left.\mathbf{X}_{\mathbf{2}}\right)$.

The molecular modeling studies generated two dimer-CYP3A4 structures that indicate that M13 or M14 formation within the active site of the enzyme is sterically feasible with minimal rearrangement of the protein. Although we do not claim that these models are definitive binding modes, they provide supportive evidence for the mechanism we propose for homodimer formation.

In summary, during the in vivo metabolite profiling of GDC-0994 in rats, we discovered two novel dimer metabolites. In vitro studies indicated these metabolites were generated across several animal species and human, with CYP3A4/5 being the most efficient. Biomimetic catalysis produced sufficient quantities of the metabolites to resolve the structures and lead to the identification of a symmetrical and asymmetric homodimer. Computational studies determine that the dimers likely form via tandem N-H abstractions from monomer GDC-0994 units and subsequent radical coupling. Molecular modeling indicates that the CYP3A4 pocket is large and flexible enough to accommodate the dimer in the active site. All together, these studies provide evidence that large dimers can be formed by $\mathrm{P} 450$ s and the mechanistic analysis can inform other drug discovery programs dealing with metabolism of similar substrates.

\section{Acknowledgments}

We thank Michael Drummond from Chemical Computing Group for sharing his knowledge and insights about structural aspects of cytochrome P450 ligand binding.

\section{Authorship Contributions}

Participated in research design: Takahashi, Grandner, Bobba, Liu, Beroza, Ma.

Conducted experiments: Takahashi, Bobba, Liu, Ma.

Contributed to computational studies: Grandner (DFT), Beroza (modeling).

Performed data interpretation: Takahashi, Grandner, Bobba, Liu, Beroza, Zhang, Ma.

Contributed to the writing of the manuscript: Takahashi, Grandner, Bobba, Liu, Beroza, Ma.

\section{References}

Becke AD (1993) Density-functional thermochemistry. III. The role of exact exchange. $J$ Chem Phys 98:5648-5652.

Belin P, Le Du MH, Fielding A, Lequin O, Jacquet M, Charbonnier JB, Lecoq A, Thai R, Courçon $\mathrm{M}$, Masson C, et al. (2009) Identification and structural basis of the reaction catalyzed by CYP121, an essential cytochrome P450 in Mycobacterium tuberculosis. Proc Natl Acad Sci USA 106:7426-7431.

Blake JF, Burkard M, Chan J, Chen H, Chou KJ, Diaz D, Dudley DA, Gaudino JJ, Gould SE, Grina J, et al. (2016) Discovery of (S)-1-(1-(4-Chloro-3-fluorophenyl)-2-hydroxyethyl)-4-(2-((1methyl-1H-pyrazol-5-yl)amino)pyrimidin-4-yl)pyridin-2(1H)-one (GDC-0994), an extracellular signal-regulated kinase 1/2 (ERK1/2) inhibitor in early clinical development. J Med Chem 59: 5650-5660.

Cerny MA (2016) Prevalence of non-cytochrome P450-mediated metabolism in food and drug administration-approved oral and intravenous drugs: 2006-2015. Drug Metab Dispos 44: 1246-1252.

Chemical Computing Group ULC (2018) Molecular Operating Environment (MOE), 2018.01, Chemical Computing Group ULC, Montreal, QC, Canada.

Chen MS and White MC (2007) A predictably selective aliphatic C-H oxidation reaction for complex molecule synthesis. Science 318:783-787.

Cusack KP, Koolman HF, Lange UE, Peltier HM, Piel I, and Vasudevan A (2013) Emerging technologies for metabolite generation and structural diversification. Bioorg Med Chem Lett 23: $5471-5483$.
Dansette PM, Bertho G, and Mansuy D (2005) First evidence that cytochrome P450 may catalyze both S-oxidation and epoxidation of thiophene derivatives. Biochem Biophys Res Commun 338: 450-455.

Davis JA, Greene RJ, Han S, Rock DA, and Wienkers LC (2011) Formation of raloxifene homodimer in CYP3A4, evidence for multi-substrate binding in a single catalytically competent $\mathrm{P} 450$ active site. Arch Biochem Biophys 513:110-118.

Emoto C, Nishida H, Hirai H, and Iwasaki K (2007) CYP3A4 and CYP3A5 catalyse the conversion of the N-methyl-D-aspartate (NMDA) antagonist CJ-036878 to two novel dimers. Xenobiotica 37:1408-1420.

Feng Y, Wang J, Liu L, and Guo Q (2003) C-H and N-H bond dissociation energies offive- and six-membered ring aromatic compounds. J Phys Org Chem 16:883-890.

Frisch MJ, Trucks GW, Schlegel HB, Scuseria GE, Robb MA, Cheeseman JR, Scalmani G, Barone V, Mennucci B, Petersson GA, et al. (2013) Gaussian 09, revision E.01, Gaussian, Inc., Wallingford, CT. Gillam EM, Notley LM, Cai H, De Voss JJ, and Guengerich FP (2000) Oxidation of indole by cytochrome P450 enzymes. Biochemistry 39:13817-13824.

Grandner JM, Cacho RA, Tang Y, and Houk KN (2016) Mechanism of the P450-catalyzed oxidative cyclization in the biosynthesis of griseofulvin. ACS Catal 6:4506-4511.

Grimme S, Ehrlich S, and Goerigk L (2011) Effect of the damping function in dispersion corrected density functional theory. J Comput Chem 32:1456-1465.

Guengerich FP and Munro AW (2013) Unusual cytochrome P450 enzymes and reactions. J Biol Chem 288: $17065-17073$

Halgren TA (1996a) Merck molecular force field. 2. MMFF94 van der Waals and electrostatic parameters for intermolecular interactions. J Comput Chem 17:520-552.

Halgren TA (1996b) Merck molecular force field. 3. Molecular geometries and vibrational frequencies for MMFF94. J Comput Chem 17:553-586.

Halgren TA (1996c) Merck molecular force field. 5. Extension of MMFF94 using experimental data, additional computational data, and empirical rules. J Comput Chem 17:616-641.

Halgren TA (1996d) Merck molecular force field. I. Basis, form, scope, parameterization, and performance of MMFF94. J Comput Chem 17:490-519.

Halgren TA (1999a) MMFF VI. MMFF94s option for energy minimization studies. J Comput Chem 20:720-729.

Halgren TA (1999b) MMFF VII. Characterization of MMFF94, MMFF94s, and other widely available force fields for conformational energies and for intermolecular-interaction energies and geometries. J Comput Chem 20:730-748.

Halgren TA and Nachbar RB (1996) Merck molecular force field. 4. Conformational energies and geometries for MMFF94. J Comput Chem 17:587-615.

Hayes C, Ansbro D, and Kontoyianni M (2014) Elucidating substrate promiscuity in the human cytochrome 3A4. J Chem Inf Model 54:857-869.

Isin EM and Guengerich FP (2007) Complex reactions catalyzed by cytochrome P450 enzymes. Biochim Biophys Acta 1770:314-329.

Kirouac DC, Schaefer G, Chan J, Merchant M, Orr C, Huang SA, Moffat J, Liu L, Gadkar K, and Ramanujan S (2017) Clinical responses to ERK inhibition in $B R A F^{\mathrm{V} 600 \mathrm{E}}$-mutant colorectal cancer predicted using a computational model. NPJ Syst Biol Appl 3:14

Lee ML, Aliagas I, Feng JA, Gabriel T, O’Donnell TJ, Sellers BD, Wiswedel B, and Gobbi A (2017) chemalot and chemalot_knime: command line programs as workflow tools for drug discovery. J Cheminform 9:38.

Makino M, Sugimoto H, Shiro Y, Asamizu S, Onaka H, and Nagano S (2007) Crystal structures and catalytic mechanism of cytochrome P450 StaP that produces the indolocarbazole skeleton. Proc Natl Acad Sci USA 104:11591-11596.

Marenich AV, Cramer CJ, and Truhlar DG (2009) Universal solvation model based on solute electron density and on a continuum model of the solvent defined by the bulk dielectric constant and atomic surface tensions. J Phys Chem B 113:6378-6396.

Ortiz de Montellano PR and De Voss JJ(2005) Substrate oxidation by cytochrome P450 enzymes, in: Cytochrome P450: Structure, Mechanism, and Biochemistry (Ortiz de Montellano PR ed) pp 183-230, Kluwer Academic/Plenum Publishers, New York.

Reilly CA, Henion F, Bugni TS, Ethirajan M, Stockmann C, Pramanik KC, Srivastava SK, and Yost GS (2013) Reactive intermediates produced from the metabolism of the vanilloid ring of capsaicinoids by p450 enzymes. Chem Res Toxicol 26:55-66.

Ren L, Grina J, Moreno D, Blake JF, Gaudino JJ, Garrey R, Metcalf AT, Burkard M, Martinson M, Rasor K, et al. (2015) Discovery of highly potent, selective, and efficacious small molecule inhibitors of ERK1/2. J Med Chem 58:1976-1991.

Ribeiro RF, Marenich AV, Cramer CJ, and Truhlar DG (2011) Use of solution-phase vibrational frequencies in continuum models for the free energy of solvation. J Phys Chem B 115:14556-14562. Schadt S, Bister B, Chowdhury SK, Funk C, Hop CECA, Humphreys WG, Igarashi F, James AD, Kagan M, Khojasteh SC, et al. (2018) A decade in the MIST: learnings from investigations of drug metabolites in drug development under the "metabolites in safety testing" regulatory guidance. Drug Metab Dispos 46:865-878.

Schrödinger LLC (2016) Schrödinger Release 2016-3: Glide, Schrödinger, LLC, New York.

Song KS, Liu L, and Guo QX (2003) Remote substituent effects on N-X (X = H, F, Cl, CH3, Li) bond dissociation energies in para-substituted anilines. J Org Chem 68:262-266.

Treiber A, Dansette PM, El Amri H, Girault J-P, Ginderow D, Mornon J-P, and Mansuy D (1997) Chemical and biological oxidation of thiophene: preparation and complete characterization of thiophene S-oxide dimers and evidence for thiophene S-oxide as an intermediate in thiophene metabolism in vivo and in vitro. $J$ Am Chem Soc 119:1565-1571.

Varga A, Soria JC, Hollebecque A, LoRusso P, Bendell J, Huang SA, Wagle MC, Okrah K, Liu L, Murray E, et al. (2020) A first-in-human phase I study to evaluate the ERK1/2 inhibitor GDC0994 in patients with advanced solid tumors. Clin Cancer Res 26:1229-1236.

Woithe K, Geib N, Zerbe K, Li DB, Heck M, Fournier-Rousset S, Meyer O, Vitali F, Matoba N, Abou-Hadeed K, et al. (2007) Oxidative phenol coupling reactions catalyzed by OxyB: a cytochrome $\mathrm{P} 450$ from the vancomycin producing organism. implications for vancomycin biosynthesis. J Am Chem Soc 129:6887-6895.

Zhao Y and Truhlar DG (2008) Computational characterization and modeling of buckyball tweezers: density functional study of concave-convex pi...pi interactions. Phys Chem Chem Phys 10:2813-2818.

Address correspondence to: Shuguang Ma, Drug Metabolism and Pharmacokinetics, Genentech, Inc., 1 DNA Way, MS 412a, South San Francisco, CA 94080. E-mail: ma.shuguang@gene.com 